\title{
The Microwave Q-branch Spectrum of Germane in the Vibrational Ground State
}

\author{
W. Stahl and H. Dreizler \\ Abteilung Chemische Physik im Institut für Physikalische Chemie der Universität Kiel \\ L. Jörissen and W. A. Kreiner \\ Abteilung Physikalische Chemie, Universität Ulm \\ Z. Naturforsch. 41 a, 747-751 (1986); received March 1, 1986 \\ We present an analysis of the rotational spectra of ${ }^{70} \mathrm{GeH}_{4},{ }^{72} \mathrm{GeH}_{4}$ and ${ }^{74} \mathrm{GeH}_{4}$ in the \\ vibrational ground state measured by microwave Fourier transform (MWFT) spectroscopy. All \\ quartic, sextic and octic tensor centrifugal distortion constants have been determined. A \\ discussion of spin-rotation and spin-spin interactions will be given in a subsequent paper.
}

\section{Introduction}

The microwave spectrum of germane, $\mathrm{GeH}_{4}$, has been a subject of investigations since many years. One reason for the great interest may be due to the tetrahedral and therefore nonpolar equilibrium structure of the molecule. Nevertheless it is possible to observe Q-branch microwave transitions because a nonzero dipole moment exists as a consequence of centrifugal distortion [1]. P- and R-branch transitions have been measured in the IR-region [2]. A great deal of theoretical work has been published in the field of tetrahedral molecules. The major part of these papers investigate the centrifugal distortion effect [3-8]. The Hamiltonian stated there allows very accurate prediction of the line frequencies if the tensor centrifugal distortion constants are known. Only one theoretical treatment of the line shift caused by the isotopic substitution of the central atom is known to the authors [9]. The order of magnitude of this effect is about $100 \mathrm{kHz}$. A further group of publications [10-16] deals with the spin-rotation and spin-spin interaction caused by the four hydrogen atoms of the tetrahedral molecule in equivalent positions. The resulting hyperfine splittings of the F-type Q-branch transitions reach values up to $100 \mathrm{kHz}$ and have also been observed by MWFT-spectroscopy.

The first microwave spectrum of germane was obtained by Kagann et al. [1] with a Stark-modula-

Reprint requests to Prof. Dr. H. Dreizler, Institut für Physikalische Chemie, Olshausenstr. 40-60, D-2300 Kiel. tion spectrometer using averaging of the spectra. A period in the order of several hours was required to obtain one averaged spectrum. The line width was about $1 \mathrm{MHz}$ (estimated from [1], Figure 1). Only those transitions (E-species) showing a linear Stark effect could be measured. Even at highest voltages the quadratic Stark effect of most of the Aand F-type transitions is too small to give a sufficient modulation. We tried to overcome this restriction by utilizing the MWFT-technique as it has been done already by Bauder's group in Zürich for the investigation of the homologes methane and silane $[17,18]$. As a result we observed lines of all symmetry species (A, E, F) quite often with very high signal-to-noise ratio and a line width of $20 \mathrm{kHz}$ within a measuring period of a few minutes. In addition the combination of a Stark cell and a MWFT-spectrometer allowed us to obtain an estimate of the magnitude of the electric dipole moment.

In this paper we intend to report on the centrifugal distortion effect for the species ${ }^{70} \mathrm{GeH}_{4},{ }^{72} \mathrm{GeH}_{4}$ and ${ }^{74} \mathrm{GeH}_{4}$ only.

\section{Experimental}

The isotopically enriched ( $>90 \%$ of the enriched species) samples of ${ }^{70} \mathrm{GeH}_{4},{ }^{72} \mathrm{GeH}_{4}$ and ${ }^{74} \mathrm{GeH}_{4}$ were prepared by R. Opferkuch, Ulm, from the respective dioxides [19]. The percentage of impurities is unknown but probably small. All spectra were recorded by MWFT-spectrometers in the J-,

0340-4811 / $86 / 0500-0747 \$ 01.30 / 0$. - Please order a reprint rather than making your own copy. 


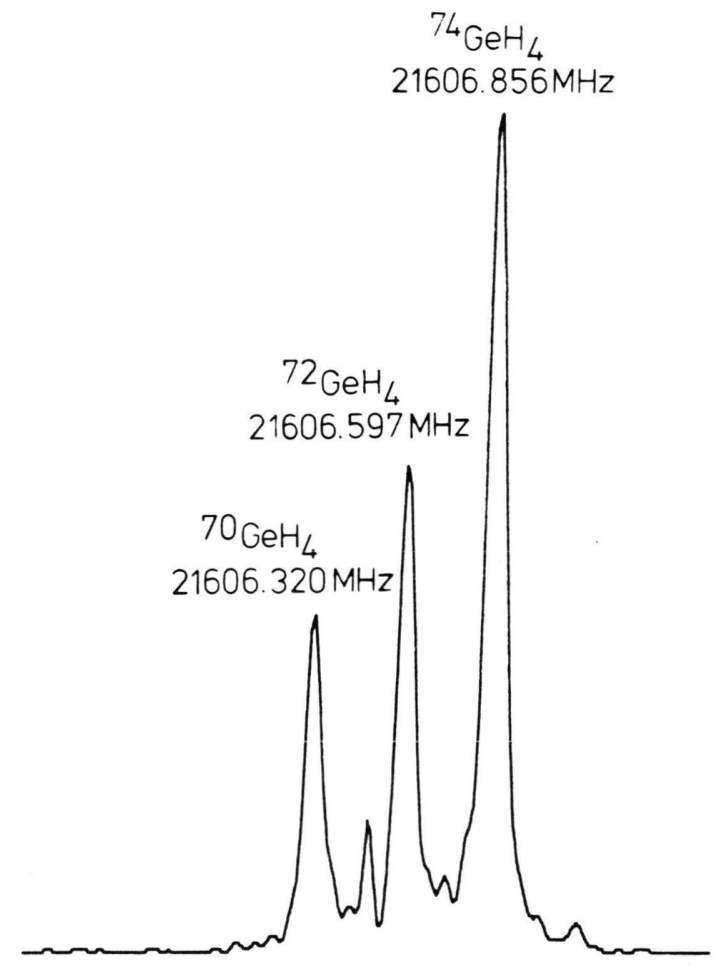

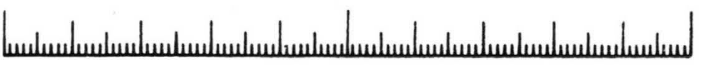

Fig. 1a. A 2-MHz-range of the rotational spectrum of a germane sample containing the isotopic species in natural abundance. The $19 \mathrm{~A}_{1}(1)-19 \mathrm{~A}_{2}(1)$ transition was measured at a pressure of $4.6 \mathrm{mTorr}$ and room temperature (about $20{ }^{\circ} \mathrm{C}$ ). Measuring time 10 minutes, 10 million averaging cycles. Pulse power about 3 Watts at TWTA output, pulse length $700 \mathrm{~ns}$. The transient emission signal was recorded with a sample interval of $20 \mathrm{~ns}$ and 1024 data points (extended with zeros to 4096 data points prior to Fourier transformation). Spectral point distance $12.5 \mathrm{kHz}$.

X-, Ku- and K-band, 5-26.4 GHz. The experimental setup has been described already earlier [20-22]. The sample pressure varied from $1 \mathrm{mTorr}$ to $20 \mathrm{mTorr}$. In the case of lines showing hyperfine structure a pressure lower than $5 \mathrm{mTorr}$ had to be used for higher resolution. All measurements have been carried out at room temperature (about $20{ }^{\circ} \mathrm{C}$ ). For MWFT-spectroscopy the intensity of the observable signal depends in a "sinusoidal" manner from the power of the polarizing pulse, from the pulse length and the dipole matrix element [23]. In our case the extreme small dipole moment of germane and the experimentally limited pulse

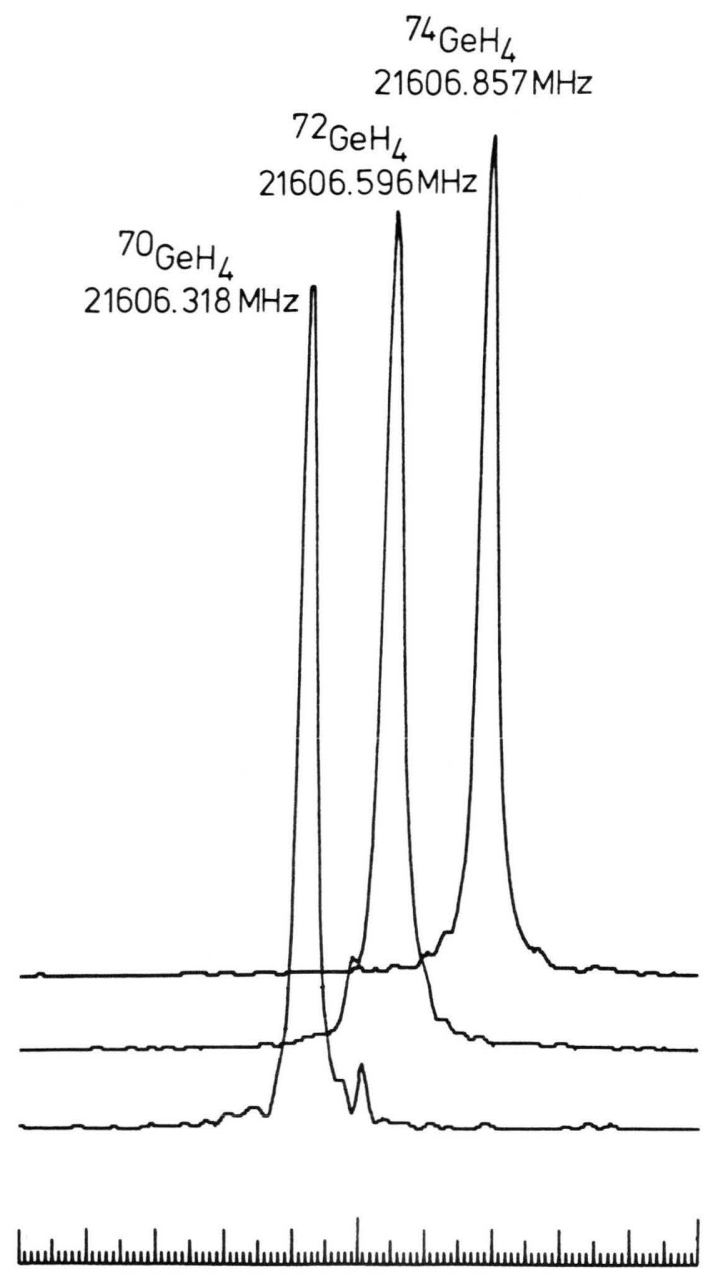

Fig. $1 \mathrm{~b}$. The $19 \mathrm{~A}_{1}(1)-19 \mathrm{~A}_{2}$ (1) transition recorded under roughly the same conditions as above. Isotopically enriched ${ }^{70} \mathrm{GeH}_{4^{-}},{ }^{72} \mathrm{GeH}_{4}$ - and ${ }^{74} \mathrm{GeH}_{4}$-samples were used.

power prevented us from reaching the optimum $\pi / 2$-condition. A pulse length longer than $700 \mathrm{~ns}$ turned out to be not useful. So we always measured at the maximum available pulse power (specifications of the TWTAs: J-band $25 \mathrm{~W}, \mathrm{X}$-band and $\mathrm{Ku}$ band $10 \mathrm{~W}, \mathrm{~K}$-band $3 \mathrm{~W}$ ) and at a constant pulse length of $700 \mathrm{~ns}$.

\section{Measurements and Analysis}

We already pointed out that a lot of theoretical contributions in the field of tetrahedral molecules 
exist. Therefore we do not intend do repeat any theoretical treatment. Nevertheless we will give all formulas used for analyzing the data.

The nomenclature used in this article for the classification of energy levels is the same as it has been used by Hougen [14, 24, 25] and other scientists [26]. Some authors use a different classfication method $[5,15,16]$.

The probably most important aspect of the analysis concerns the evaluation of the tensorial centrifugal distortion parameters. The frequencies of the measured lines used for this purpose are given in Table 1. The data were weighted as the inverse squares of the estimated experimental errors. In general the F-species transitions have a higher uncertainty than the other lines because the hyperfine splittings prevented an accurate determination of the center frequency. All data were obtained from measurements on the isotopically enriched samples. When we used a sample of natural isotopic composition $\left({ }^{70} \mathrm{GeH}_{4}: \quad 20.52 \%\right.$, ${ }^{72} \mathrm{GeH}_{4}: 27.43 \%,{ }^{73} \mathrm{GeH}_{4}: \quad 7.76 \%,{ }^{74} \mathrm{GeH}_{4}: 36.54 \%$, ${ }^{76} \mathrm{GeH}_{4}: 7.76 \%$ ) in earlier stages of our experiment we often observed weak and superimposed lines. In order to illustrate the shift caused by isotopic substitution of the central atom a spectrum of the natural isotopic mixture and, for comparison, the spectra of the isotopically enriched samples are shown in Figure 1.

For the evaluation of the tensor centrifugal distortion constants the following Hamiltonian [26] was used

$$
\begin{aligned}
H= & H_{\mathrm{S}}+H_{\mathrm{T}}, \\
H_{\mathrm{S}}= & B_{0} J^{2}-D_{\mathrm{S}} J^{4}+H_{\mathrm{S}} J^{6}+L_{\mathrm{S}} J^{8}, \\
H_{\mathrm{T}}= & {\left[D_{\mathrm{T}}+H_{4 \mathrm{~T}} J^{2}+L_{4 \mathrm{~T}} J^{4}\right] \Omega_{4} } \\
& +\left[H_{6 \mathrm{~T}}+L_{6 \mathrm{~T}} J^{2}\right] \Omega_{6}+L_{8 \mathrm{~T}} \Omega_{8}
\end{aligned}
$$

with $H_{\mathrm{S}}$ being the scalar and $H_{\mathrm{T}}$ being the tensorial part. $J$ is the operator of the angular momentum, $B_{0}$ the rotational constant, $D_{\mathrm{S}}, H_{\mathrm{S}}$ and $L_{\mathrm{S}}$ the scalar centrifugal distortion constants, and $\Omega_{k}, k=4,6,8$, are the $k$-th rank tensor operators for the tetrahedral molecule. The respective tensor centrifugal distortion parameters $D_{\mathrm{T}}, H_{4 \mathrm{~T}}, L_{4 \mathrm{~T}}, H_{6 \mathrm{~T}}, L_{6 \mathrm{~T}}$ and $L_{8 \mathrm{~T}}$ were determined by fitting the exprimental line frequencies by the fitting procedure XY4FIT [27] in interaction with the program XY4TOP [27] which calculates the energy levels of tetrahedral molecules by direct diagonalization of (1). The results are
Table 1. Measured lines of ${ }^{70} \mathrm{GeH}_{4},{ }^{72} \mathrm{GeH}_{4}$ and ${ }^{74} \mathrm{GeH}_{4}$. In the case of lines showing hyperfine splittings a center frequency was estimated. Del (in $\mathrm{kHz}$ ) $=$ Observed frequency (in $\mathrm{MHz}$ ) - Calculated frequency (in $\mathrm{MHz}$ ). The columns designated by " $E$ " indicate the estimated error:

\begin{tabular}{|c|c|c|c|c|c|c|c|c|c|}
\hline & & & \multicolumn{3}{|l|}{${ }^{70} \mathrm{GeH}_{4}$} & \multicolumn{2}{|l|}{${ }^{72} \mathrm{GeH}_{4}$} & \multicolumn{2}{|l|}{${ }^{74} \mathrm{GeH}_{4}$} \\
\hline & & & Obs. & Del $E$ & E & Obs. & Del $E$ & Obs. & Del $E$ \\
\hline 19 & $A 2(2)$ & A1 & 5043.181 & $-4 A$ & & 5043.167 & $-1 A$ & 5043.155 & $+5 A$ \\
\hline 14 & $\mathrm{~F} 2(3)$ & $\mathrm{F} 1(1)$ & 5405.398 & -130 & C & 5405.465 & -20 & 5405.505 & $-16 c$ \\
\hline & $E(2)$ & $E(1)$ & 5774.185 & & & 5774.279 & & 5774.369 & \\
\hline & $F 1(4)$ & FeC & 5854.347 & +210 & & & & 5854.559 & $+12 \mathrm{c}$ \\
\hline 17 & $E(3)$ & $E(2)$ & 6722.723 & $-\theta A$ & & 6722.896 & $4 A$ & 6723.056 & $-13 \mathrm{~B}$ \\
\hline 17 & $F 1(4)$ & $\mathrm{F} 2(2)$ & 7208.776 & -3 & & 7208.848 & -40 & 7200.921 & $-7 \mathrm{c}$ \\
\hline 15 & $E(2)$ & $E(1)$ & 7314 & $-6 F$ & & 7314.925 & $-3 A$ & 7314.995 & $-6 A$ \\
\hline 15 & $F 1(3)$ & $F 2(2)$ & 7822.174 & & & 7822.258 & -40 & 7822.338 & $-9 B$ \\
\hline 14 & A1 & $A 2$ & 8920.171 & - & A & 8920.249 & $3 \mathrm{~A}$ & 8920.323 & $-5 A$ \\
\hline 20 & $\mathrm{~F} 2(4)$ & $F 1(3)$ & & & & 9488.933 & +20 & 9489.065 & $+11 \mathrm{C}$ \\
\hline 16 & & & & & & 951 & $-3 A$ & 9510.709 & $-8 \mathrm{~B}$ \\
\hline 14 & $F 1(2)$ & $\mathrm{FeC1}$ & & & & 9553.143 & & 9553.232 & $=$ \\
\hline 18 & $E(3)$ & $E(2)$ & 9615.170 & $-1 F$ & A & 9615.271 & $+4 \mathrm{~A}$ & 9615.363 & $+1 \mathrm{~A}$ \\
\hline 16 & $\mathrm{~F} 2(3)$ & F1(2) & 9678.941 & -15 & & 9679.042 & $-6 \mathrm{~B}$ & 9679.124 & $-50 \mathrm{~B}$ \\
\hline 14 & $E(2)$ & $E(1)$ & 9950.363 & $O E$ & & 9950.472 & & 9950.575 & $+\angle A$ \\
\hline 18 & $\mathrm{~F} 2(4)$ & $F 1(2)$ & 10575.173 & -10 & & 10575.304 & $3 \mathrm{c}$ & C $\quad 10575.427$ & \\
\hline 16 & F1(3) & $F 2(1)$ & 10654.795 & & & 10654.913 & $3 \mathrm{C}$ & C 10655.027 & $-2 c$ \\
\hline & A1 & $A 2(1)$ & 10939.187 & $+3 f$ & & 10939.374 & & A 10939.551 & -3 \\
\hline 15 & $F 1(4)$ & $F 2(2)$ & & & & 11184.648 & -18 & & \\
\hline 17 & AL & A1 & 11358.388 & - & A & 11358.457 & & A 11358.524 & $2 \mathrm{~A}$ \\
\hline 15 & F1(2) & $F 2(1)$ & 11692.413 & $+1 \mathrm{E}$ & & 11692.510 & + & B 11692.606 & 2 \\
\hline 15 & $\mathrm{~F} 2(3)$ & $F 1(1)$ & 12554.018 & $+4 \mathrm{~F}$ & & & & 12554.270 & 1 \\
\hline 17 & $F 2(4)$ & $F 1(3)$ & 12630.416 & -410 & c & 126 & +20 & C 12630.990 & 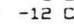 \\
\hline 19 & $\mathrm{~F} 1(4)$ & $F 2(3)$ & 12690.721 & $+13 \mathrm{c}$ & & 12690.854 & +20 & C 12690.982 & $-1 c$ \\
\hline 17 & $\mathrm{~F} 2(3)$ & $F 1(2)$ & 12892.492 & & & 12492.667 & +460 & c $\quad 12892.757$ & + \\
\hline 20 & $A 1(2)$ & $A E$ & 13576.386 & - & A & 13576.408 & $-50 \mathrm{k}$ & $8 \quad 13576.427$ & ¿ \\
\hline 17 & $E(2)$ & $E(1)$ & 13709.253 & 0 A & A & 13709.412 & $+7 \mathrm{~B}$ & 13709.560 & -1 A \\
\hline & $F 2(4)$ & $F 1(2)$ & 14380.518 & +110 & & 14380.670 & -530 & C 14380.894 & $+12 \mathrm{c}$ \\
\hline 16 & $E(2)$ & $E(1)$ & 14691.156 & & A & 14691.274 & +90 & $=14691.386$ & $+4 A$ \\
\hline 18 & $\mathrm{~F} 2(3)$ & $F 1(1)$ & 14895.916 & +18 & c & 14895.989 & $-3 c$ & C 14896.101 & \\
\hline 16 & $\mathrm{~F} 2(2)$ & F1(1) & 15002.545 & $+1 E$ & & 15002.674 & +90 & 15002.793 & $0 \mathrm{~B}$ \\
\hline 16 & $A 2$ & A1 (1) & 15905.511 & +56 & & 15905.677 & $+9 B$ & B 15905.843 & $+5 A$ \\
\hline 20 & $F 1(4)$ & $F 2(2)$ & 17027.095 & $+11 \mathrm{E}$ & & 17027.316 & & $=17027.534$ & +16 \\
\hline 18 & $F 1(3)$ & $F 2(2)$ & 17058.566 & +120 & C & 17058.760 & +9 & & \\
\hline 19 & F1(3) & Fe(2) & & & & 17073.711 & $-8 c$ & $=192$ & $+10 \mathrm{C}$ \\
\hline 19 & A1 & $A D(1)$ & 21606.318 & + & & 21606.597 & +1 & 21606.459 & \\
\hline 18 & $A 1(1)$ & $A 2(1)$ & 21741.058 & & & 21741.188 & & 21741.313 & \\
\hline 18 & $E(2)$ & $E(1)$ & 55.522 & & & & & & \\
\hline
\end{tabular}
$\mathrm{A}=10 \mathrm{kHz}, \mathrm{B}=50 \mathrm{kHz}, \mathrm{C}=100 \mathrm{kHz}$. Both, experimental errors and uncertainties in the determination of the center frequency of weak or split lines are included.

summarized in Table 2. All errors are standard errors and are given in units of the least significant digit. The correlation matrices are shown in Table 3.

Comparing the centrifugal distortion parameters of the three isotopic species one observes that the standard errors are too large to show an exact isotopic dependence. According to Watson's theory [9] the constants $B_{0}, D_{\mathrm{S}}$ and $D_{\mathrm{T}}$ should be independent of the central mass $m_{x}$, whereas the quartic and sextic centrifugal distortion constants should be affected as indicated in the following formula [9]:

$\Delta H_{4 \mathrm{~T}}=(7 / 88) \Delta H$,

$\Delta H_{6 \mathrm{~T}}=-(15 / 22) \Delta H$,

$$
\begin{aligned}
\Delta H & =-\left(\frac{256}{35}\right)\left[\frac{m_{y} \Delta m_{x}}{m_{x}\left(M+\Delta m_{x}\right)}\right] B_{0}^{5} \\
& \cdot \zeta_{23}^{2} \zeta_{24}^{2}\left(\frac{1}{\omega_{3}^{2}}-\frac{1}{\omega_{4}^{2}}\right)^{2} .
\end{aligned}
$$


Table 2. Tensor centrifugal distortion constants. Standard errors in parentheses (in units of the least significant digit). All data are to be multiplied by a factor given in the last column. The last two lines show the number of fitted lines and the standard deviation of the fit.

\begin{tabular}{lcccl}
\hline & ${ }^{70} \mathrm{GeH}_{4}$ & ${ }^{72} \mathrm{GeH}_{4}$ & ${ }^{74} \mathrm{GeH}_{4}$ & \\
\hline$D_{\mathrm{T}}$ & $6.777466(14)$ & $6.7775865(99)$ & $6.777647(10)$ & $10^{-2} \mathrm{MHz}$ \\
$H_{4 \mathrm{~T}}$ & $-5.38483(89)$ & $-5.38735(68)$ & $-5.38545(69)$ & $10^{-6} \mathrm{MHz}$ \\
$H_{6 \mathrm{~T}}$ & $2.96868(27)$ & $2.97188(20)$ & $2.97435(24)$ & $10^{-6} \mathrm{MHz}$ \\
$L_{4 \mathrm{~T}}$ & $4.033(14)$ & $4.071(11)$ & $4.031(11)$ & $10^{-10} \mathrm{MHz}$ \\
$L_{6 \mathrm{~T}}$ & $-4.2363(79)$ & $-4.2518(63)$ & $-4.2451(71)$ & $10^{-10} \mathrm{MHz}$ \\
$L_{8 \mathrm{~T}}$ & $-7.908(13)$ & $-7.912(10)$ & $-7.922(13)$ & $10^{-10} \mathrm{MHz}$ \\
Lines & 33 & 35 & 35 & \\
Standard & 10.7 & 10.9 & 8.4 & $\mathrm{kHz}$ \\
deviation & & & & \\
\hline
\end{tabular}

Table 3. Correlation matrices. All correlation coefficients are multiplied by 1000 .

\begin{tabular}{crrrrrr}
\hline${ }^{70} \mathrm{GeH}_{4}$ & \multicolumn{1}{c}{$D_{\mathrm{T}}$} & \multicolumn{1}{c}{$H_{4 \mathrm{~T}}$} & \multicolumn{1}{c}{$H_{6 \mathrm{~T}}$} & $L_{4 \mathrm{~T}}$ & $L_{6 \mathrm{~T}}$ & $L_{8 \mathrm{~T}}$ \\
$D_{\mathrm{T}}$ & 1000 & & & & & \\
$H_{4 \mathrm{~T}}$ & -994 & 1000 & & & & \\
$H_{6 \mathrm{~T}}$ & -27 & 77 & 1000 & & & \\
$L_{4 \mathrm{~T}}$ & 979 & -995 & -119 & 1000 & & \\
$L_{6 \mathrm{~T}}$ & 29 & -79 & -989 & 125 & 1000 & \\
$L_{8 \mathrm{~T}}$ & -464 & 472 & -413 & -472 & 425 & 1000 \\
\hline${ }^{72} \mathrm{GeH}_{4}$ & \multicolumn{1}{c}{$D_{\mathrm{T}}$} & \multicolumn{1}{c}{$H_{4 \mathrm{~T}}$} & $H_{6 \mathrm{~T}}$ & $L_{4 \mathrm{~T}}$ & \multicolumn{1}{c}{$L_{6 \mathrm{~T}}$} & $L_{8 \mathrm{~T}}$ \\
$D_{\mathrm{T}}$ & 1000 & & & & & \\
$H_{4 \mathrm{~T}}$ & -994 & 1000 & & & & \\
$H_{6 \mathrm{~T}}$ & 276 & -272 & 1000 & & & \\
$L_{4 \mathrm{~T}}$ & 982 & -996 & 262 & 1000 & & \\
$L_{6 \mathrm{~T}}$ & -292 & 288 & -990 & -276 & 1000 & \\
$L_{8 \mathrm{~T}}$ & -171 & 172 & -298 & -173 & 284 & 1000 \\
\hline${ }^{74} \mathrm{GeH}_{4}$ & $D_{\mathrm{T}}$ & $H_{4 \mathrm{~T}}$ & $H_{6 \mathrm{~T}}$ & $L_{4 \mathrm{~T}}$ & $L_{6 \mathrm{~T}}$ & $L_{8 \mathrm{~T}}$ \\
$D_{\mathrm{T}}$ & 1000 & & & & & \\
$H_{4 \mathrm{~T}}$ & -992 & 1000 & & & & \\
$H_{6 \mathrm{~T}}$ & -218 & 264 & 1000 & & & \\
$L_{4 \mathrm{~T}}$ & 976 & -995 & -295 & 1000 & & \\
$L_{6 \mathrm{~T}}$ & 200 & -245 & -986 & 279 & 1000 & \\
$L_{8 \mathrm{~T}}$ & -328 & 344 & -374 & -355 & 420 & 1000 \\
\hline
\end{tabular}

$m_{x}$ and $m_{y}$ are the masses of the central atom $(\mathrm{Ge})$ and the ligands $(H) . \Delta m_{x}$ indicates the change of the central mass when the central atom is substituted, $\Delta H_{4 \mathrm{~T}}$ and $\Delta H_{6 \mathrm{~T}}$ are the respective changes of the quartic and sextic centrifugal distortion constants. The quantities $\zeta_{23}$ and $\zeta_{24}$ are associated with the diagonal Coriolis constants $\zeta_{3}$ and $\zeta_{4}$ by

$$
\zeta_{23}^{2}=(2 / 3)\left(1-\zeta_{3}\right), \quad \zeta_{24}^{2}=(2 / 3)\left(1-\zeta_{4}\right)
$$

and $\omega_{3}$ and $\omega_{4}$ are the normal frequencies expressed in $\mathrm{cm}^{-1}$. Evaluation of the expressions $(2 \mathrm{a}-\mathrm{c})$ shows that the predicted changes in $H_{4} \mathrm{~T}$ and $H_{6 \mathrm{~T}}$ are smaller than the experimental error limits. An explanation of the significantly observed shift of $D_{\mathrm{T}}$ is neither given by Watson's theory nor has it been described elsewhere.

Watson's theory applies to the equilibrium configuration of the molecule while observation deals with the vibrational ground state. This may account for discrepancies between calculated and observed isotope effects. For example, the $B_{\mathrm{e}}$ constant must be the same for all isotopic species under consideration, but the $B_{0}$ values as well as the vibrational frequencies are likely to be slightly different, similar to methane $\left({ }^{12} \mathrm{CD}_{4}-{ }^{13} \mathrm{CD}_{4}\right.$ or $\left.{ }^{12} \mathrm{CH}_{4}-{ }^{13} \mathrm{CH}_{4}\right)[28,29]$; taking Hecht's expression [3] for $D_{\mathrm{T}}$,

$$
D_{\mathrm{T}}=\frac{1}{5}\left(\frac{1}{\omega_{2}^{2}}-\frac{\zeta_{23}^{2}}{\omega_{3}^{2}}-\frac{\zeta_{24}^{2}}{\omega_{4}^{2}}\right) B^{5}
$$

one expects a small effect even for the $D_{\mathrm{T}}$ constant.

The accurate data obtained from the measurements on the isotopic species of germane show that the MWFT spectroscopy is a very useful tool for the investigation of molecules with small dipole moments. An improvement of the centrifugal distortion constants will be possible when more lines in higher microwave bands can be measured. We believe that an extension of our MWFT spectrometer to the $\mathrm{V}$ band (26 to $40 \mathrm{GHz}$ ) is possible.

\section{Acknowledgements}

The authors wish to thank all members of the Kiel microwave group. We are especially indebted to Jon T. Hougen from the National Bureau of Standards in Washington for a discussion about the symmetry classification of tetrahedral molecules, R. Opferkuch from the University of Ulm for the preparation of various germane samples from germanium 
dioxide and A. Bauder and M. Oldani from the ETH Zürich for providing us with a computer program. We further thank the Deutsche For-

[1] R. H. Kagann, I. Ozier, G. A. McRae, Can. J. Phys. 57, 593 (1979).

[2] I. Ozier and A. Rosenberg, Can. J. Phys. 51, 1882 (1973).

[3] K. T. Hecht, J. Mol. Spectrosc. 5, 355 (1960).

[4] K. T. Hecht, J. Mol. Spectrosc. 5, 390 (1960).

[5] J. Moret-Bailly, J. Mol. Spectrosc. 15, 344 (1965)

[6] J. K. G. Watson. J. Mol. Spectrosc. 40, 536 (1971).

[7] A. J. Dorney and J. K. G. Watson, J. Mol. Spectrosc. 42, 135 (1972).

[8] S. M. Kirschner and J. K. G. Watson, J. Mol. Spectrosc. 47, 347 (1973).

[9] J. K. Watson, J. Mol. Spectrosc. 74, 483 (1979).

[10] R. F. Curl, Jr., J. V. V. Kasper, and K. S. Pitzer, J. Chem. Phys. 46, 3220 (1966).

[11] P.-N. Yi, I. Ozier, and C. H. Anderson, Phys. Rev. $165,92(1967)$.

[12] I. Ozier, L. M. Crapo, and S. S. Lee, Phys. Rev. 172, 63 (1968).

[13] P.-N. Yi, I. Ozier, and N. F. Ramsey, J. Chem. Phys. 55, $5215(1971)$.

[14] J. T. Hougen, J. Mol. Spectrosc. 46, 490 (1973).

[15] F. Michelot, B. Bobin, and J. Moret-Bailly, J. Mol. Spectrosc. 76, 374 (1979).

[16] F. Michelot, J. Mol. Spectrosc. 106, 77 (1984). schungsgemeinschaft and Fonds der Chemie for funds. All calculations are carried out at the Rechenzentrum of the University of Kiel.

[17] M. Oldani, M. Andrist, and A. Bauder, J. Mol. Spectrosc. 110, 93 (1985).

[18] M. Oldani, Dissertation ETH No. 7720, Zürich 1985.

[19] W. A. Kreiner, R. Opferkuch, A. G. Robiette, and P. H. Turner, J. Mol. Spectrosc. 85, 442 (1981).

[20] G. Bestmann, H. Dreizler, E. Fliege, and W. Stahl, J. Mol. Struct. 97, 215 (1983).

[21] G. Bestmann and H. Dreizler, Z. Naturforsch. 37a, 58 (1982).

[22] W. Stahl, G. Bestmann, H. Dreizler, U. Andresen, and R. Schwarz, Rev. Sci. Instrum. 56(9), 1759 (1985).

[23] J. C. McGurk, H. Mäder, R. T. Hofmann, T. G. Schmalz, and W. H. Flygare, J. Chem. Phys. 61, 3759 (1974).

[24] J. T. Hougen, J. Chem. Phys. 37, 1433 (1962).

[25] J. T. Hougen, J. Chem. Phys. 39, 358 (1963).

[26] J. Ozier, M. C. L. Gerry, and A. G. Robiette, J. Phys. Chem. Ref. Data 10, 1085 (1981).

[27] The computer programs XY4TOP and XY4FIT were obtained from A. Bauder and M. Oldani, ETH Zürich.

[28] D. L. Gray and A. G. Robiette, Mol. Phys. 37, 1901 (1979).

[29] W. A. Kreiner, P. Müller, L. Jörissen, M. Oldani, and A. Bauder, to be published. 